\title{
Behandlungsmethoden bei Milchstau (übervolle, harte, schmerzhafte Brüste) bei stillenden Müttern
}

Zakarija-Grkovic I, Stewart F. Treatments for breast engorgement during lactation. Cochrane Database of Systematic Reviews 2020, Issue 9. Art. No.: CD006946. DOI: 10.1002/14651858.CD006946.pub4.

\section{Worum geht es?}

Ein Milchstau entsteht durch einen Überschuss an Milch, der zu geschwollenen, harten und schmerzhaften Brüsten führt. Stauungen treten häufiger auf, wenn das Stillen nach Zeitplan durchgeführt wird, wenn Frauen Schwierigkeiten beim Stillen haben oder sie von ihren Babys getrennt werden. Dies führt dazu, dass die Brüste nicht ausreichend entleert werden.

\section{Warum ist das wichtig?}

Ein Milchstau ist belastend und führt zu Komplikationen wie Entzündungen der Brust, wunden/rissigen Brustwarzen und einer verminderten Milchversorgung. In der Folge kann es dazu kommen, dass Frauen das Stillen einstellen. Es mangelt an beständiger Evidenz für wirksame Behandlungsformen.

\section{Welche Evidenz haben wir gefunden?}

Für diese Aktualisierung haben wir (am 2. Oktober 2019) nach Studien gesucht, die sich mit der Behandlung von Milchstau bei stillenden Frauen befassen. Wir fanden 21 Studien mit 2.170 Frauen und 17 verschiedenen Interventionen.

Bei Brustschmerzen sind kalte Kohlblätter möglicherweise besser als die Routineversorgung oder kalte Gel-Packs. Es ist unklar, ob kalte Kohlblätter besser sind als Kohlblätter bei Raumtemperatur, oder Kohlblätter bei Raumtemperatur besser sind als Wärmflaschen, oder eine Kohlblattextrakt-Creme besser ist als eine PlaceboCreme, weil die Vertrauenswürdigkeit der Evidenz niedrig war. Bezogen auf die Brusthärte sind kalte Kohlblätter möglicherweise besser als die Routineversorgung, aber es ist unklar, ob sie besser sind als kalte Gel-Packs. Bei Milchstau sind Kohlblätter bei Raumtemperatur möglicherweise besser als Wärmflaschen. Es ist unklar, ob eine Kohlblattextrakt-Creme besser ist als eine Placebo-Creme, weil die Vertrauenswürdigkeit der Evidenz niedrig war.

Bei Brustschmerzen ist eine Kräuterkompresse möglicherweise besser als eine heiße Kompresse, und eine Massagetherapie plus Kaktus-|Aloe-Kompresse möglicherweise besser als eine Massagetherapie allein. Es ist unklar, ob eine Kaktus-/AloeKompresse besser ist als eine Massagetherapie, da die Vertrauenswürdigkeit der Evidenz niedrig war. Eine Kaktus-/AloeKompresse ist möglicherweise im Vergleich zu einer Massagetherapie besser bei Brusthärte. Massage plus Kaktus-|Aloe-Kältekompresse ist möglicherweise besser bei Brusthärte als Massage allein. Die Auswirkungen der Kompressen-Behandlungen auf den Milchstau und das Abstillen sind unklar, da die Vertrauenswürdigkeit der Evidenz sehr niedrig war.

Protease wirkt möglicherweise besser bei Brustschmerzen und Brustschwellungen, während Serrapeptase im Vergleich zu Placebo möglicherweise besser bei Milchstau wirkt. Es ist unklar, ob Serrapeptase Brustschmerzen oder -schwellungen reduziert oder ob Oxytocin den Milchstau im Vergleich zu Placebo reduziert, da die Vertrauenswürdigkeit der Evidenz niedrig war.

Bei Brustschmerzen ist die Wirksamkeit von kalten Gel-Packs im Vergleich zu Kontrollbehandlungen unklar, da die Vertrauenswürdigkeit der Evidenz niedrig war. Bei Brusthärte sind kalte Gel-Packs möglicherweise besser als die Routineversorgung. Es ist unklar, ob mehr Frauen nach einer Behandlung mit kalten Gel-Packs im
Vergleich zur Routineversorgung mit dem Stillen aufhören, weil die Vertrauenswürdigkeit der Evidenz niedrig war.

Was die Meinung der Frauen zur Behandlung anbelangt, so war die Vertrauenswürdigkeit der Evidenz niedrig. Mehr Frauen waren mit kalten Kohlblättern zufrieden als mit der Routineversorgung oder mit kalten Gel-Packs. Möglicherweise gibt es kaum Unterschiede in der Zufriedenheit der Frauen zwischen kalten Gel-Packs und Routineversorgung.

Drei Studien berichteten über unerwünschte Ereignisse. In keiner der Gruppen, die Medikamente erhielten, traten unerwünschte Ereignisse auf (niedrige Vertrauenswürdigkeit der Evidenz), und 2/250 Frauen, die mit Kräuterkompressen behandelt wurden, erlitten Hautreizungen im Vergleich zu 0/250 Frauen in der Gruppe mit heißen Kompressen (moderate Vertrauenswürdigkeit der Evidenz).

\section{Was bedeutet das?}

Es gibt Hinweise darauf, dass einige Behandlungsmaßnahmen bei Milchstau vielversprechend sein könnten, z. B. Kohlblätter, kalte Gel-Packs, Kräuterkompressen und Massagen, aber es sind weitere Studien erforderlich, um die tatsächliche Wirkung dieser Interventionen zu ermitteln.

Bibliografie

Die Hebamme 2020; 33: 10 DOI 10.1055/a-1284-3014 ISSN 0932-8122

C 2020. Thieme. All rights reserved. Georg Thieme Verlag KG, Rüdigerstraße 14, 70469 Stuttgart, Germany' 